\title{
TRAFFIC SIMULATION MODEL AND ITS APPLICATION FOR ESTIMATING SATURATION FLOW AT SIGNALISED INTERSECTION
}

\author{
Sheela Alex ${ }^{1}$, Kuncheria P Isaac ${ }^{2}$ \\ ${ }^{1}$ College of Engineering, Trivandrum, India \\ ${ }^{2}$ All India Council for Technical Education, New Delhi, India
}

Received 10 February 2014; accepted 8 July 2014

\begin{abstract}
The efficiency of a transportation network depends upon the performance characteristics of its various components. To improve the efficiency, proper planning and design of various facilities have to be implemented. For this, simulation models seem to be more powerful than analytical procedure. This paper focuses on simulating traffic movements at signalised intersections by developing a micro simulation model using a high level programming language. The developed model TRAFFICSIM, which is highly user friendly, is capable of simulating any traffic movements at various geometric as well as signal phasing characteristics. TRAFFICSIM is a true replication of the original traffic scenario as it is capable of updating the acceleration, lateral and linear spacing as well as deceleration characteristics which is highly influenced by the traffic behaviour and signal phasing. The validated model is then applied to determine the saturation flow models during $100 \%$ car only condition as well as mixed traffic conditions. The saturation flow model developed under $100 \%$ car only condition is $s=522 w$, where $s$ is the saturation flow in $\mathrm{pc} / \mathrm{hr}$ and $w$ is the carriageway width in $\mathrm{m}$. The saturation flow obtained under $100 \%$ car only both in homogeneous condition and in a mixed traffic condition is $1911 \mathrm{pc} / \mathrm{hr}$ and $1908 \mathrm{pc} / \mathrm{hr}$ respectively which shows close agreement with the HCM values.
\end{abstract}

Keywords: mixed traffic, simulation model, saturation flow, signalised intersection.

\section{Introduction}

Signalised intersections are one of the key locations in any transportation network, hence improper planning of the intersection affects the performance and productivity of the whole road network. For this, a thorough analysis has to be made in order to utilise the signalised intersections effectively. The analytical approach has the limitation of underlying assumption of homogeneity, which is having high variations of traffic characteristics when compared with a mixed traffic condition. Simulation models on the other hand, follow the dynamic nature of the traffic system, and give a continuous view of the state of the traffic system over time. This characteristic of simulation model is an advantage over analytical models, since it gives more information and insight to what is happening in the system in detail. Hence, researchers concentrated on appropriate modelling technique and the modelling approach through simulation, which is emerged as the most powerful, flexible and acceptable solution searching tool. Review of the literature shows that though many simulation studies were done to analyse traffic flow at midblock sections, only a few studies have been done

${ }^{1}$ Corresponding author: sheelaalex@cet.ac.in 
to develop a model to simulate traffic flow at signalised intersections under non lane based heterogeneous traffic conditions.

This paper focuses on simulating traffic movements at signalised intersections by developing a micro simulation model using a high level programming language. In order to develop a model for a signalised intersection of varying geometry, due consideration has to be given to represent the geometrical features. A four arm intersection is the most commonly occurring type of intersections. The width of the approach also varies from single lane to multilane. In the present study, a four arm intersection is proposed to be simulated with varying approach width. The simulated model is then adapted to the field conditions available in Indian cities. The simulation model consists of various component models such as headway generation, speed generation, acceleration characteristics, vehicle movement and vehicle accumulation, intersection characteristics, and signal characteristics. These component models were integrated to form the newly developed TRAFFICSIM model. The developed model is then applied to estimate the saturation flow at various traffic combinations.

\section{Review}

Computer simulation is a valuable tool for the analysis and design of complex transportation systems. Simulation models may be classified as being static or dynamic, deterministic or stochastic, and discrete or continuous. Keller and Saklas (1984) developed a procedure to estimate Passenger Car Equivalent (PCE) values for large vehicles moving over an urban network using TRANSYT simulation model. IRC SP: 41 had also recommended tentative PCU values for conversion of different types of vehicles into equivalent PCU. Banks et al.
(2000) mentioned the choice of whether to use a discrete or continuous simulation model is a function of the characteristics of the system and the objectives of the study. Most of the simulation works were done to develop Passenger Car Unit values for the vehicles in the mixed traffic conditions for urban roads. Hossain (2001) used micro simulation technique to model traffic operations at signalised intersections of developing cities like Dhaka, the capital of Bangladesh. Arasan and Koshy (2005) developed a simulation model for urban roads. Mallikarjuna and Rao (2006) developed a Cellular Automata based simulation model, to estimate PCU under different traffic regimes. Arasan and Krishnamurthy (2008) conducted a study on the effect of traffic volume and road width on PCU values of vehicles using Microscopic simulation at mid-block sections of urban roads. Arasan and Vedagiri (2006) applied the simulation model to estimate the saturation flow rate of heterogeneous traffic. Gowri and Sivanandan (2008) developed a simulation model and examined the effects of left turn channelisation on vehicle waiting times. Arasan and Arkatkar (2010), Arasan and Dhivya (2010) also have developed microsimulation models for midblock sections. Radhakrishnan and Mathew (2011) developed a traffic simulation model integrating the concepts of cellular automata and driver- vehicle objects.

\section{Simulation Frame Work}

Traffic simulation models require randomness of various events which is to be incorporated to take care of the stochasticity in vehicle arrivals and other traffic flow aspects. The sequence of random numbers can be generated by various means. The master flow chart for the developed simulation model is shown in Fig. 1. 


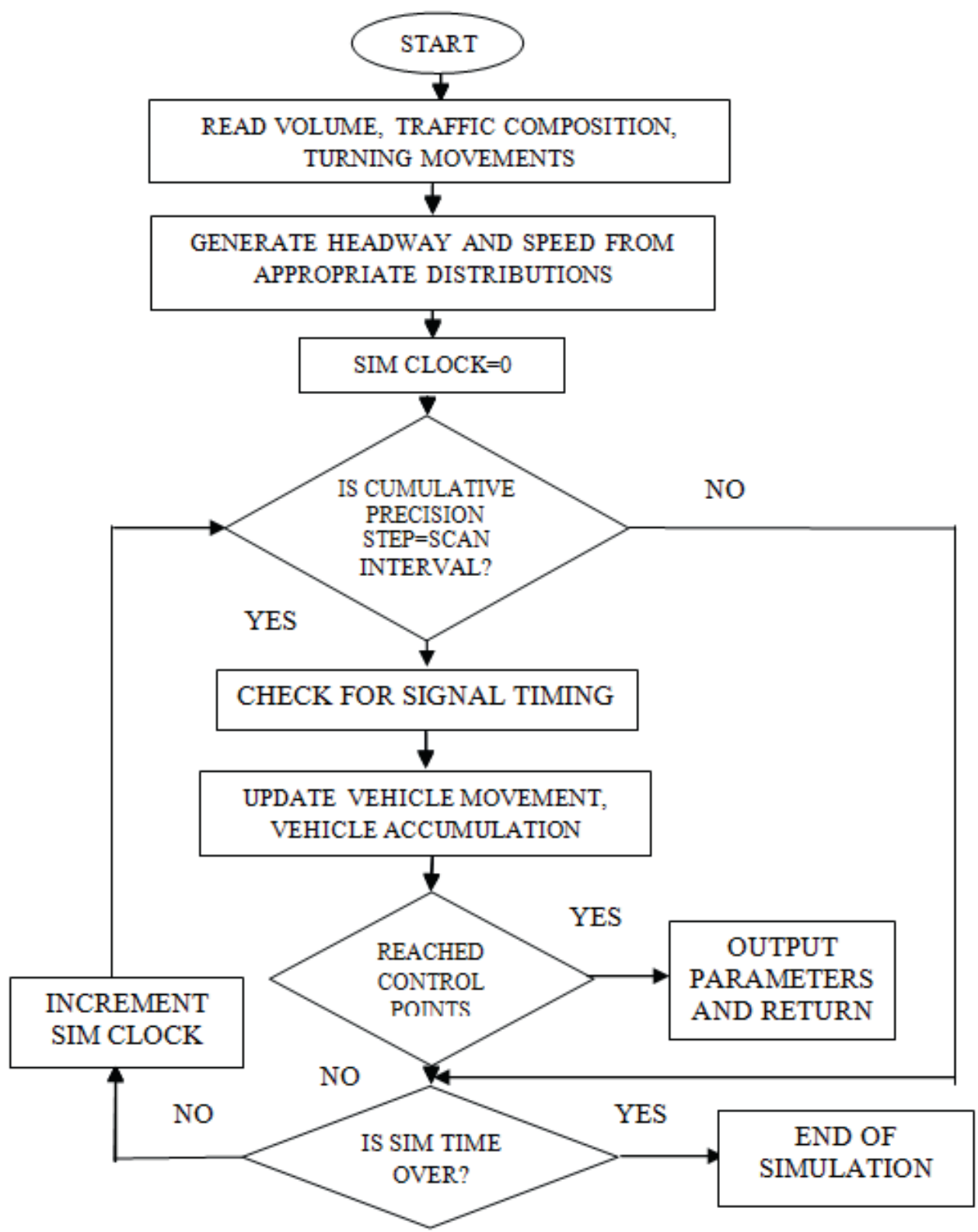

Fig. 1.

Master Flow Chart for TRAFFICSIM 
The simulation procedure applied for this study contains four different random number seed files containing sufficient number of seeds for the generation of additional random number streams for replication of simulation runs. These are used as inputs to model the specific features of an intersection, which are following some particular statistical distribution. In this study, a fixed increment time advance approach was used. In this approach, the simulation clock is advanced in increments of exactly one second. After each update of the clock, a check is made to determine whether any event has occurred during the previous interval of length one second. At higher traffic flow, there is a chance of more vehicle arrivals during each scan interval of one second. To deal with this aspect, an additional clock for scanning with a precision of eight milliseconds, for updating the state of the system as the simulation progresses is provided.

The present study is concentrated on the behaviour of vehicles at intersections, so the movement of vehicle along the approaches of the intersection for a length of $260 \mathrm{~m}$, were analysed. A mean speed is assigned using normal distribution to each vehicle based on the vehicle type. Once it enters into the network, the vehicle adjusts its speed based on the time headway to the vehicle in front and also based on the basic equations of motion. A check is also made to test whether the generated speeds are within the maximum specified limit or not. If the generated speed is less than or equal to the maximum, the speed is assigned. If not, a new mean speed is assigned, using the same process with a fresh set of random numbers. An initial acceleration rate of zero $\mathrm{m} / \mathrm{s}^{2}$ is assigned to the vehicles at the time of entering the network. Once the vehicle enters the network, acceleration at any point was considered to be a linear variable with speed as referred in Traffic flow theory and Control by Drew (1968) as given in Eq. (1):

$\frac{d u}{d t}=\alpha-\beta v$

where,

$\alpha$ is the maximum possible vehicle

acceleration, $\mathrm{m} / \mathrm{s}^{2}$,

$\beta=$ constant having units ${ }^{-1}$.

Isaac (1995) presented a set of values for the variables as shown in Table 1 . These values were incorporated in the model to obtain the acceleration values for each speed.

\section{Table 1}

a, $\beta$ Values for Different Classes of Vehicles

\begin{tabular}{|l|l|l|l|}
\hline Type of vehicle & $\alpha$ & $\beta$ & $\alpha / \beta$ \\
\hline car & 1.75 & 0.087 & 20.36 \\
\hline two_wheeler & 1.09 & 0.060 & 18.36 \\
\hline bus & 0.64 & 0.040 & 16.24 \\
\hline three_wheeler & 1.01 & 0.076 & 13.29 \\
\hline
\end{tabular}

Source: Isaac (1995)

In order to attain the random nature of traffic, the percentage composition of the vehicles present in the system varies in each signal cycle. Provisions are given in the model to input varying composition of vehicles. Vehicles are generated at the entry point of the simulation road stretch based on its composition. Even at the entry point of the simulation stretch, the direction of movement are assigned based on the percentage turning movements along left, straight and right. A uniformly distributed random number is generated and is compared with the cumulative 
percentage of turning movements of each type of vehicle, in the ascending order. When the cumulative turning percentage of a particular movement is less than the random number, and that of the other movement is greater, the latter is adopted as the required movement for that particular vehicle type.

As the work is concentrated on the traffic behaviour at signalised intersections, out of the total $260 \mathrm{~m}$ of the simulation stretch, the length of the study approach is taken as 100 $\mathrm{m}$ till the stop line, depending on the turning behaviour, the vehicles are placed at the entry of the simulation stretch. The approach is provided with lanes of known width assigned for different turning movements. The left turning vehicles are placed at the left lane of the simulation stretch. Similarly, right turning vehicles are placed at the right lane of the approach and straight vehicles are placed at the middle portion of the approach.
In the case of first vehicle, the vehicle can attain its desired speed and move along the simulation stretch, as free vehicle entering into the empty simulation stretch. With the arrival of the next vehicle, if the front vehicle is slow compared to that of following vehicle, the sequence of checking the available lateral and linear clearances is to be made. The gaps available in the system at every time step are informed to the vehicle object. For this purpose scanning is done on the left, right and also on the front side of the vehicle. If the gaps are insufficient for the vehicle overtaking, the vehicle is kept in queue and the checks for vehicle movement are again made in the next scan interval. Linear and lateral spacing models developed in the previous studies were incorporated in the model. Isaac (1995) developed relationship for the linear and lateral spacing of the current vehicle based on the speed of the subject vehicle as explained in Table 2 and Table 3.

Table 2

Relationships for Linear Space Gaps with Respect to Speed

\begin{tabular}{|c|c|c|}
\hline Vehicle under consideration & Vehicle in front & Relation \\
\hline Car & Car & SP $=1.1008 \exp \left(0.0558^{*}\right.$ speed $)$ \\
\hline Car & Three-wheeler & SP $=0.898 \exp \left(0.0609^{*}\right.$ speed $)$ \\
\hline Car & Two-wheeler & SP $=1.1301 \exp \left(0.0532^{*}\right.$ speed $)$ \\
\hline Three-wheeler & Bus & SP $=1.576 \exp \left(0.0437^{*}\right.$ speed $)$ \\
\hline Three-wheeler & Car & SP $=1.0885 \exp \left(0.0495^{*}\right.$ speed $)$ \\
\hline Three-wheeler & Two-wheeler & SP $=0.9335 \exp \left(0.0513^{*}\right.$ speed $)$ \\
\hline Three-wheeler & Three-wheeler & SP $=1.0369 \exp \left(0.0497^{*}\right.$ speed $)$ \\
\hline Two-wheeler & Two-wheeler & SP $=0.8650 \exp \left(0.0524^{*}\right.$ speed $)$ \\
\hline Two-wheeler & Car & SP $=1.2091 \exp \left(0.0452^{*}\right.$ speed $)$ \\
\hline Two-wheeler & Bus & SP $=0.9296 \exp \left(0.0535^{*}\right.$ speed $)$ \\
\hline Two-wheeler & Three-wheeler & $\mathrm{SP}=0.9100 \exp \left(0.060^{*}\right.$ speed $)$ \\
\hline Bus & Bus & $\mathrm{SP}=1.7795 \exp \left(0.0512^{*}\right.$ speed $)$ \\
\hline Bus & Three-wheeler & $\mathrm{SP}=1.7163 \exp \left(0.0484^{*}\right.$ speed $)$ \\
\hline Bus & Car & $\mathrm{SP}=1.831 \exp \left(0.0497^{*}\right.$ speed $)$ \\
\hline Bus & Two-wheeler & $\mathrm{SP}=1.5000 \exp \left(0.050^{*}\right.$ speed $)$ \\
\hline
\end{tabular}

$S P$ - linear spacing between the vehicles, $m$

Source: Isaac (1995) 
Table 3

Relationships with Speed for Lateral Space Gaps

\begin{tabular}{|c|c|c|}
\hline Vehicle under consideration & Vehicle in the side & Relation \\
\hline Car & Car & LSP $=0.70+0.01($ speed $)$ \\
\hline Car & Three-wheeler & $\mathrm{LSP}=0.62+0.012($ speed $)$ \\
\hline Car & Two-wheeler & $\mathrm{LSP}=0.47+0.013($ speed $)$ \\
\hline Car & Bus & $\mathrm{LSP}=0.605+0.012($ speed $)$ \\
\hline Three-wheeler & Two-wheeler & $\mathrm{LSP}=0.52+0.014($ speed $)$ \\
\hline Three-wheeler & Bus & $\mathrm{LSP}=0.59+0.020($ speed $)$ \\
\hline Three-wheeler & Three-wheeler & $\mathrm{LSP}=0.45+0.015$ (speed) \\
\hline Two-wheeler & Two-wheeler & $\mathrm{LSP}=0.57+0.016($ speed $)$ \\
\hline Two-wheeler & Bus & $\mathrm{LSP}=0.62+0.011($ speed $)$ \\
\hline Bus & Bus & $\mathrm{LSP}=0.43+0.020($ speed $)$ \\
\hline
\end{tabular}

LSP - lateral spacing between the vehicles, $m$

Source: Isaac (1995)

The lateral spacing relationships are applied only at the initial portion of the vehicle movement after it was being generated and also for the vehicles entering the section during the green phase. During the signal change from green to amber and to red, the vehicle reduces its speed and they tried to occupy the positions with a minimum spacing between the vehicles depending upon the vehicle type. The same will be the case when vehicles start dissipating during the green phase. Table 4 shows the minimum clearance thresholds for different categories of vehicles. Knowing the availability of the longitudinal and lateral spacing, the vehicles are assumed to occupy any lateral position of the approach.

\section{Table 4}

Lateral Clearance Thresholds for Different Categories of Vehicles

\begin{tabular}{|c|c|}
\hline Vehicle Type & Minimum clearance $(\mathbf{m})$ at zero speed \\
\hline Bus & 0.30 \\
\hline Car & 0.30 \\
\hline Three_wheeler & 0.20 \\
\hline Two_wheeler & 0.10 \\
\hline
\end{tabular}

Source: Arasan and Koshy (2005)

During each scan interval, the positions of all the vehicles in the system are updated. The program updates the status of all vehicles in the list, picking them one by one, starting from the entry end of the simulation stretch. While updating the position of vehicles, the vehicle also has to check for the signal phase. Based on the corresponding signal, the vehicles are acted upon accordingly.
Whenever a vehicle approaching the intersection facing amber phase, it tries to decelerate depending upon its position from the stop line. When the vehicle reaches the intersection, it has to check the signal phasing for straight and right turning movements. When the signal phase is amber/red, the vehicle approaching the intersection is assigned with a deceleration rate as suggested by Gates et al. (2007) as given in Eq. (2): 
decelerationrate $=0.51 *$ speed $-0.05 *$ distance $+2.7 *$ brakeresponsetime

where, distance is the distance of the vehicle from the stop line, speed is the speed of the vehicle and brake response time is assumed as $1 \mathrm{~s}$.

Once the leading vehicle decides to stop, the following vehicles will respond to the stopping behaviour as per car following model and comes to a stop and join the queue. As free left is permitted in the model, this is applicable only to non-left turning vehicles. Whenever the signal is red at an intersection, the vehicles of the traffic stream accumulate at the approach road by making use of the availability of gaps and type of turning movement. Full consideration has been given in the model for the optimum utilisation of the approach by the vehicles when forming a queue. Hence, the narrower vehicles can use the intervehicular space to move towards the front of the queue. Whenever the signal changes from red to green, vehicles start dissipating from the stop line. The leading vehicle in the queue will start to move with the respective initial acceleration rate. The position of all the vehicles is updated during dissipation using equations of motion for each and every scan interval.

\section{Object Oriented Methodology for Simulation Modelling}

For modelling the heterogeneous traffic flow along an intersection, an object oriented methodology for microscopic simulation was implemented. Object oriented programming (OOP) language has gained recognition in the development of scientific codes and the programmer produces more comprehensible, maintainable and flexible codes with less effort and expense using powerful and flexible characteristics of OOP.
The simulation software developed for this study TRAFFICSIM was written using C\# programming language. Most of the literature related to the present study is available for homogeneous traffic conditions using OOP concepts. It is difficult to predict the versatility of these models in a heterogeneous, non-lane based traffic flow at signalised intersections. It is the most suitable programming language having object oriented features like static constructors, properties, delegates and events etc.

\subsection{Functions and their Descriptions}

The computer program developed in this software incorporates various functions in order to represent the road system, manage the vehicle movements as well as controlling the signal system. The main functions and their descriptions are given under this section.

In TRAFFICSIM, the vehicles are modelled as objects. Each vehicle is assigned with a detection number, vehicle ID. Each vehicle type is declared, where four types of vehicle, two wheeler, three wheeler, car and bus are returned and are represented by different color. Each vehicle type has unique member data (length, breadth, mean speed, standard deviation of speed, turning radius etc.).

Data variables like lateral and longitudinal spacing are assigned with values based on their vehicular interactions like car-car, car-bus etc. as mentioned in Table 2 and Table 3. A function was defined in the model to manage the movement of entire traffic stream at the intersection. Each vehicle type is assigned a mean speed and at every position the speed of the vehicle is checked and is not allowed to exceed beyond the maximum speed limit. The position of 
each vehicle type is updated based on the equations of motion for each and every scan interval. The model gets the current vehicle details from the array. It invokes the two functions to move the vehicles and to overtake the vehicles by checking the available space ahead for updating the vehicle position as explained in section 3. If space is insufficient to move forward, the function calls upon the vehicle to invoke the process of overtaking logic. If this is not possible, the vehicle adjusts its speed and follows the front vehicle. Once the process has been done, the vehicle details are updated in the output file. As the vehicle approaches the intersection, and when the signal is on amber/red position, the vehicle is assigned with a deceleration value which depends on the vehicle speed and the distance from the stop line at the intersection as mentioned in Eq. (2). At every time step, the vehicle details such as current position, gap available, speed, acceleration characteristics, its distance from the stop line, deceleration rate and its nature of movements are updated, and thus each vehicle object is associated with a driver behaviour in this study, thus forming a driver-vehicle entity.

The capability of a simulation tool to represent varying geometric characteristics and signal phases is important in establishing the performance of the intersection. The most important aspect is to accurately represent the geometry defined by the number of approaches, width of each approach, width of each lane specified for the turning movements and the turning space (the space required for each turning movement in the intersection). For the vehicle to turn on the respective direction, the coordinates rx, ry, tx, ty and theta (angle of turn) values were obtained based on the position of vehicles.
The system consists of a road with clear carriageway of any desired width. The entire road space is considered as a single unit and the vehicles are represented as rectangular blocks on the road space. The road width is an input variable in the model, which can have any value greater than 1.2 $\mathrm{m}$, which is just required for a two wheeler only condition. When the traffic composition considered includes buses, a minimum road of single lane width of $3.1 \mathrm{~m}$ may be given as the input. The model accurately represents the geometry defined by the number of approaches, width of each approach and the space occupied by each turning movements in the intersection. Each road class is further composed of lanes of various widths for different turning movements. In order to represent the non lane based approach, providing the lane width as fractions of the available road width or providing as a single lane having an overall width of the road. The length of the road stretch is also a variable in the model. In the simulation model, there is no maximum limit for the length of the road. As the present study is concentrated on the intersection area, the total length of the simulation stretch was taken as 260 $\mathrm{m}$. Along the approach road, control lines known as markers are provided at an interval of $50 \mathrm{~m}$ for getting the vehicle details like vehicle type, time at which vehicle crosses the reference line and number of vehicles crossing the reference line. In order to get the accumulated vehicle count during the red phase, regions are provided along the simulation stretch at an interval of $10 \mathrm{~m}$. The interval between the regions as well as between the markers can be given as input to the model.

The signal clock is adjusted with that of the simulation clock, as the simulation clock sets to start, the signal clock also starts 
simultaneously. The cycle time, green time, double green time (both green for straight and right turning movements) and red time is assigned to each approach of the four legged intersection. The signal system is provided on all the four approaches. Double green is set up for permitting the traffic movements along the straight and right turning simultaneously. The signal system works on all the approaches based on the phase diagrams drawn for the entire intersections.

\subsection{Inputs to the Model}

To start the simulation, the global variables must be assigned with the initial values. The simulation clock, signal timer and all the other counters must be initially set to zero. The traffic signals were set to their first phases. The input parameters were provided through a $x \mathrm{ml}$ configuration file, which allows for easy data inputs to the simulation model. An extra advantage of the $\mathrm{xml}$ format is the fact that the structure of the files is determined by a published document type definition. This allows other users to read and interpret as well as write the information correctly and makes it possible to transform the input file to other formats.

The inputs to a simulation model can be classified into two types. The values of the variables which are to be varied for conducting experiments with the model and the other model parameters which do not change frequently. The user of the simulation program will have to define a certain traffic scenario over a specified roadway section by giving the value of input variables. The input file of this simulation program will contain the following variables which can be varied during model application:
1. Traffic volume on all the four approaches in vehicles per hour;

2. Traffic composition in percentage of each category of vehicle in the stream on all the four approaches;

3. Percentage movements of traffic in each lane for left, straight and right movements on all the four approaches;

4. Road length in metres;

5. Road width in metres;

6. Width split ratio, i.e. width of lanes for left turning, straight and right turning movements;

7. Mean and Standard Deviation of speeds of each category vehicle in each direction of road like South North (SN), North South (NS), East West (EW), West East (WE);

8. Acceleration characteristics of each category vehicle in each direction $(a=a$ $\beta v$ ), i.e. $\alpha$ and $\beta$ values for each category in each approach;

9. Total Simulation time;

10. Total Signal Cycle time;

11. Signal timing for double green (green for both straight and right), green, amber and red;

12. Seed number which is to be changed for replication of simulation run;

13. Location of control points (markers) for speed data;

14. Location of stretches (regions) for vehicle accumulation data;

15. Field length (length of simulation stretch);

16. Field height (breadth of simulation stretch);

17. Angle of turn at the junctions.

Built in model parameters, which are included in the input file, if required can be changed, are:

18. Details for statistical distributions for headway generation and speed generation; 
19. Reaction time of drivers in seconds;

20. Acceleration model for each category of vehicles at different speed ranges in $\mathrm{m} / \mathrm{s}^{2}$;

21. Deceleration model for the vehicle on the onset of amber/red;

22. Dimensions of each category vehicles in metres;

23. Lateral clearances of different categories of vehicle interactions;

24. Longitudinal spacing between various categories of vehicle interactions.

\subsection{Outputs of the Model}

The outputs of the model are written in files. Provisions were given to define any number of reference lines, to obtain the speed data of various vehicle types crossing the control points and also the location of stretches to obtain the vehicle accumulation data. Facilities were also given to define two points along the simulation stretch, to determine the occupancy time, i.e. the time taken by the vehicle to cover these two well defined points.

Outputs obtained are of two types:

1. Numerical values that provide detailed quantitative descriptions of the various traffic system parameters of concern, and

2. Animation of the traffic flow showing the dynamics in the state of the system during the simulation run.

Various output files which are separately generated for all the four approaches are:

1. Headway distribution file which gives the details of vehicle type and headways generated at the entry point of the simulation stretch;
2. Entry marker and exit marker file which gives the category wise details of all the vehicle types and the speed of the vehicles crossing the control points;

3. Entry region and exit region file which gives the number and type of vehicles accumulated along a certain stretch of the approach;

4. Travel time file which gives the details of the time taken by the vehicles to cover a particular length of the simulation stretch;

5. Queuelength file which gives the number of vehicles occupying the particular approach during the red phase;

6. Summary file which gives the position of all the vehicles in the simulation stretch at each scan interval;

7. Saturation flow obtained at various approaches.

The simulation model contains an animation module which provides a graphical display of the movement of vehicles when traffic flow at intersections are simulated. There is also a provision for switching the module off, for faster execution of simulation runs. The other features include:

1. A display of simulation clock time and cycle time;

2. A dynamic display of the total numbers of each category of vehicles crossing the marker 1, i.e. at the stop line;

3. A dynamic display of the number of vehicles present in the approach at any particular instant.

Fig. 2 shows the snapshot of the animation screen at the intersection area. 


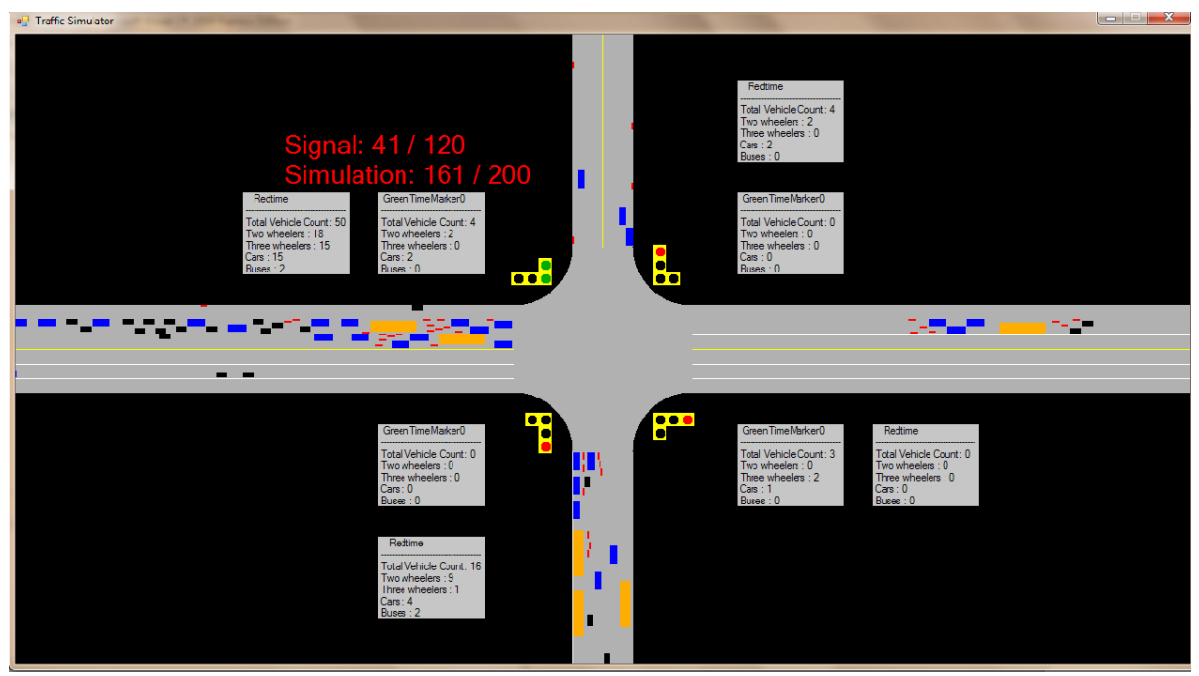

Fig. 2.

Animation Screen of the Simulation Model, TRAFFICSIM

\section{Data Collection}

The simulation model developed in the present study is to capture the varying mixed traffic conditions prevalent in India. Hence it was decided to select a few intersections of a metropolitan city of India and selected Chennai, a metro city close to Thiruvananthapuram. The intersections selected are two signalised intersections at Chennai such as:

Intersection 1 - Thiruvanmiyur signalised intersection, Chennai,

Intersection 2 - Nandanam signalised intersection, Chennai.

Data were collected by continuously video capturing traffic at signalised points for varying durations. Duration of data collection was suitably selected in order to collect sufficient number of data sets from various signal cycles. Fig. 3 shows the snapshot of Thiruvanmizhur intersection at Chennai.

Data were extracted from the video, with the help of programs which were developed using MATLAB. The extracted data were then analysed which were used for the validation of the developed model. Data collected includes the geometric characteristics, signal timings, number of vehicles discharged during green time, number of vehicles accumulated during red phase, time taken by the vehicles to cover a particular stretch of the intersection. 


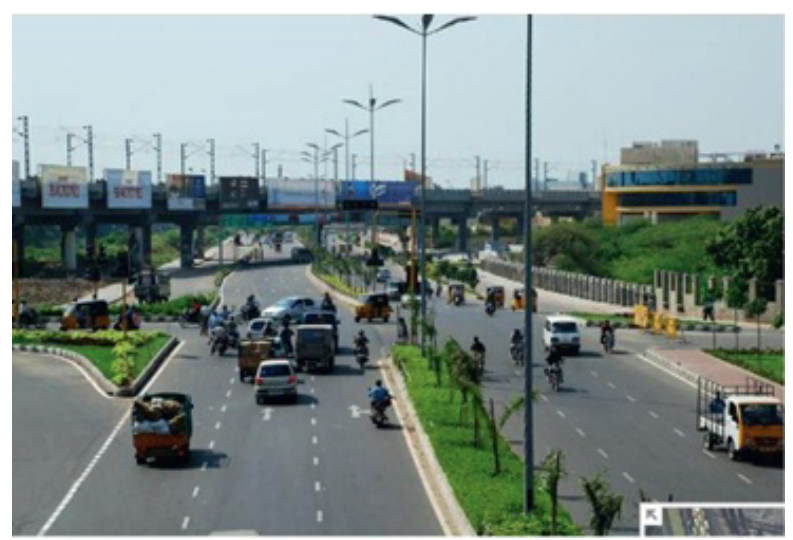

Fig. 3.

Snapshot of Thiruvanmizhur Signalised Intersection, Chennai

\subsection{Model Calibration and Validation}

In order to verify the calibration of the developed model, simulation runs were made on TRAFFICSIM. The input parameters were given similar to that of the field conditions of Thiruvanmizhur, one of the selected intersections. One of the approaches of the intersection was considered and the generated headways were analysed. Fig. 4 shows the headway distribution pattern of the vehicles generated and shows that it follows a negative exponential distribution. Also, as the vehicle approaches the intersection, it was found from animation screen that the movement of vehicles are in compliance with the traffic signal and when the signal changes to red, speed reduces to zero, and the vehicle starts accumulating.

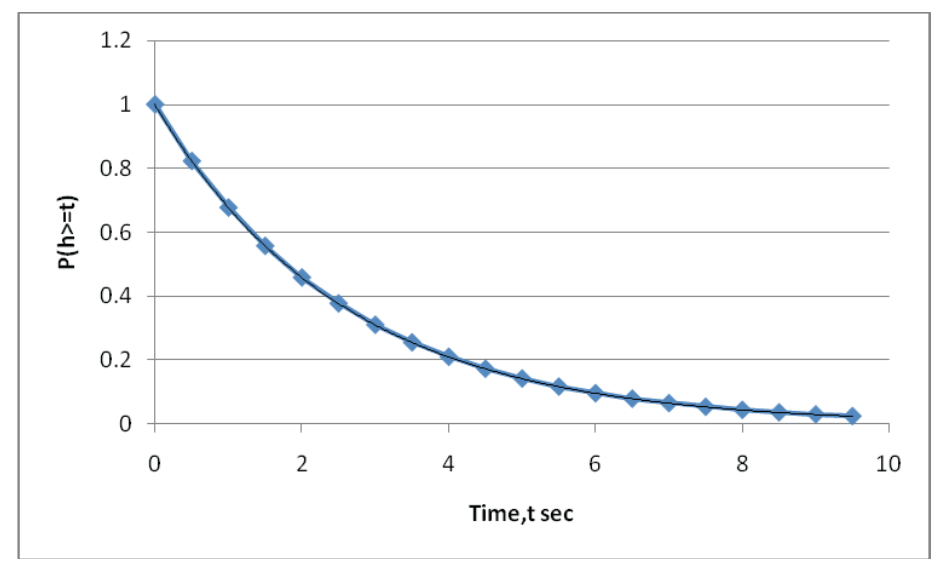

Fig. 4.

Negative Exponential Headway Distribution Graph 
The simulation model for intersection was validated based on parameters like traffic composition, speed of all vehicle types, vehicle accumulation during red phase and number of vehicles dissipated during green phase. Since the traffic on Indian roads is of heterogeneous nature, the parameters to be validated were found out in terms of number of vehicles per hour. Data collected from two four legged intersection, one at Thiruvanmizhur and one at Nandanam at Chennai were used for validation purposes. The parameters such as approach width, lane width, traffic volume, traffic composition, proportion of turning movements, mean speed of vehicles were given as inputs to the simulation model.
For Thiruvanmizhur intersection, the approach width is $12.5 \mathrm{~m}$ with the lane width for through movements as $9.52 \mathrm{~m}$, traffic volume is $2990 \mathrm{veh} / \mathrm{hr}$, percentage composition of two_wheeler, three_wheeler, car and bus are 44, 8, 39 and 9 respectively. Proportions of turning movements are left, straight and right movements as $10 \%, 85 \%$ and $5 \%$ respectively. Table 5 shows the comparison of the observed and simulated traffic composition of through vehicles at Thiruvanmizhur intersection.

In order to check the significance of the model, the MAPE value is calculated using the Eq. (3):

MAPE $=\frac{100}{n} * A B S(($ Actual - Simulated $) /$ Actual $)$

Table 5

Comparison of Observed and Simulated Traffic Composition at Thiruvanmizhur Intersection

\begin{tabular}{|c|c|c|c|c|}
\hline \multirow{2}{*}{ Vehicle type } & \multicolumn{2}{|c|}{ Average traffic composition /cycle } & \multirow{2}{*}{$\begin{array}{c}\text { ABS((Actual-sim)/ } \\
\text { actual })\end{array}$} & MAPE \\
\cline { 2 - 4 } & Actual & Simulated & 0.01 & \multirow{2}{*}{$15.52<20 \%$} \\
\hline two_wheeler & 44.97 & 45.28 & 0.23 & 0.04 \\
\hline three_wheeler & 12.13 & 9.28 & 0.34 & \\
\hline car & 39.59 & 41 & 4.42 & 0.34 \\
\hline
\end{tabular}

Since the obtained MAPE value is $15.52 \%$, which is less than $20 \%$, the model is considered to be statistically significant.
Table 6 shows the comparison of observed and simulated average speed of vehicles discharged during the green phase.

Table 6

Comparison of Observed and Simulated Average Speeds at Thiruvanmizhur Intersection

\begin{tabular}{|c|c|c|c|c|}
\hline \multirow{2}{*}{ Vehicle type } & \multicolumn{2}{|c|}{ Average speeds/cycle } & \multirow{2}{*}{$\begin{array}{c}\text { ABS } \\
\text { (Actual-sim)/actual }\end{array}$} & MAPE \\
\cline { 2 - 4 } & Actual & Simulated & 0.12 & \multirow{2}{*}{$12.05<20 \%$} \\
\hline two_wheeler & 26.85 & 23.62 & 0.11 & 0.14 \\
\hline three_wheeler & 26.46 & 23.62 & 0.12 & \\
\hline car & 27.38 & 23.62 & 22 & \\
\hline bus & 24.92 & & \\
\hline
\end{tabular}


MAPE value calculated shows significant results which indicates that the model is capable of generating speeds similar to that of the field. Table 7 shows the comparison of observed discharge flow values and those predicted by the simulated model.

\section{Table 7}

Comparison of Observed and Simulated Number of Discharged Vehicles at Thiruvanmizhur Intersection

\begin{tabular}{|c|c|c|c|c|c|}
\hline \multirow{2}{*}{ Cycle } & \multicolumn{2}{|c|}{ No. of vehicles dissipated } & Chi square & \multirow{2}{*}{ ABS((actual-sim)/actual) } & MAPE \\
\cline { 2 - 5 } & Simulated Ej & Actual Oj & $(\mathbf{O j}-\mathbf{E j}) \mathbf{2} / \mathbf{E j}$ & 0.33 \\
\hline 1 & 48 & 36 & 4.00 & 0.14 \\
\hline 2 & 50 & 44 & 0.82 & 0.04 \\
\hline 3 & 52 & 50 & 0.08 & 0.04 \\
\hline 4 & 53 & 51 & 0.08 & 0.21 \\
\hline 5 & 63 & 52 & 2.33 & 0.12 \\
\hline 6 & 66 & 59 & 0.83 & 0.08 \\
\hline 7 & 67 & 62 & 0.40 & 0.02 \\
\hline 8 & 67 & 66 & 0.02 & 0.03 \\
\hline 9 & 68 & 70 & 0.06 & 0.04 \\
\hline 10 & 68 & 71 & 0.13 & 0.05 \\
\hline 11 & 69 & 73 & 0.22 & 0.04 \\
\hline 12 & 70 & 73 & 0.12 & 0.07 \\
\hline 13 & 71 & 76 & 0.33 & \\
\hline & & & 14.51 & \\
\hline
\end{tabular}

Since the calculated Chi square value is 14.5 which is less than the critical table value of 30.14 for a level of significance 5\% and degree of freedom 12, the obtained model is statistically significant. Since the obtained MAPE value is $9.74 \%$, which is less than $10 \%$, the model is considered to be absolutely good.

To obtain the queue density at an intersection approach, 95 sq.m of area on the approach (9.5 m width of the approach and $10 \mathrm{~m}$ length) was considered in the model. The queue density, i.e. the vehicle accumulation (veh/95 sq.m) was determined by counting the number of vehicles occupying the area, when the signal was red. Statistical hypothesis test result reveals that there is no significant difference at 5\% level of significance, between the observed and simulated values of queue density. Table 8 shows the comparison of observed accumulated values and those predicted by the model. 
Table 8

Comparison of Observed and Simulated Accumulated Vehicles at Thiruvanmizhur Intersection

\begin{tabular}{|c|c|c|c|c|c|}
\hline Cycle & $\begin{array}{c}\text { Simulated } \\
(\text { veh/cycle })\end{array}$ & $\begin{array}{c}\text { Observed } \\
(\mathbf{v e h} / \mathbf{c y c l e})\end{array}$ & $\begin{array}{c}\text { Chi square } \\
(\mathbf{O j}-\mathbf{E j}) \mathbf{2} / \mathbf{E j}\end{array}$ & ABS((Actual-sim)/actual) \\
\hline 1 & 6 & 6 & 0 & 0 \\
\hline 2 & 7 & 6 & 0.143 & 0.167 \\
\hline 3 & 7 & 6 & 0.143 & 0.167 \\
\hline 4 & 8 & 6 & 0.5 & 0.333 \\
\hline 5 & 8 & 7 & 0.125 & 0.143 \\
\hline 6 & 9 & 7 & 0.444 & 0.286 \\
\hline 7 & 9 & 7 & 0.444 & 0.25 \\
\hline 8 & 10 & 8 & 0.4 & 0.25 \\
\hline 9 & 10 & 8 & 0.4 & 0.375 \\
\hline 10 & 10 & 8 & 0.818 & 0.222 \\
\hline 11 & 11 & 9 & 0.364 & 0.222 \\
\hline 12 & 11 & 9 & 0.364 & 0.2 \\
\hline 13 & 12 & 11 & 0.333 & $\mathbf{5 . 5 9 1}$ & \\
\hline Total & & & & \\
\hline
\end{tabular}

Since the MAPE value $20.5 \%$, the model is considered to be good. Also, the calculated Chi square value is 5.59, which shows that the model is statistically significant. Thus the model gets validated for traffic composition, average speeds of vehicle types, discharged vehicles as well as for the accumulated vehicles during red phase.

\section{Model Application}

The model is experimented for both single vehicle only condition and for mixed traffic condition. In the case of single vehicle only condition, there is no variation in the operating speeds, size and driving characteristics of vehicles. But in a mixed traffic situation, the vehicles of all sizes and shapes interact on the roadway with different operating characteristics and speeds, makes the traffic system more dynamic in nature.
Hence, in this study, the saturation flow was determined separately considering both the situations.

\section{1. Determination of Saturation Flow}

\section{i) Car only condition}

The developed model, TRAFFICSIM, was first applied to simulate a $100 \%$ passenger cars only traffic condition in one direction, in a four legged signalised intersection. Simulation runs were made for varying road widths having maximum input volume. The simulation time was fixed as one hour. The experimentation was done for widths of 3.5 , 4.0, 4.5, 5.5, 6.5, 7.0, 8.5, 9.5 and $10.5 \mathrm{~m}$. The signal cycle time was assumed as $120 \mathrm{~s}$ (green $56 \mathrm{~s}$, amber $4 \mathrm{~s}$, red $60 \mathrm{~s}$ ). The parameter selected to compare the results with the Webster's model, is the saturation flow, as 
it is a basic parameter to analyse the capacity of an intersection. Hence only the straight movements of traffic were considered for the analysis. The obtained saturation flow for varying lane widths in a $100 \%$ passenger car only traffic condition from the model as well as obtained by Webster and Cobbe (1966) are given in Table 9 for comparison.

Table 9

Comparison of Obtained Saturation Flow with Webster Model Values

\begin{tabular}{|c|c|c|}
\hline $\begin{array}{c}\text { Lane width } \\
(\mathbf{m})\end{array}$ & $\begin{array}{c}\text { Saturation Flow(passenger cars/h) from } \\
\text { the model }\end{array}$ & $\begin{array}{c}\text { Saturation Flow obtained by Webster and } \\
\text { Cobbe (1966) (pcu/h) }\end{array}$ \\
\hline 3.5 & 1929 & 1890 \\
\hline 4.5 & 3086 & 2250 \\
\hline 5.5 & 3141 & 2900 \\
\hline 6.5 & 3214 & 3412 \\
\hline 7 & 3563 & 3675 \\
\hline 8 & 3793 & 4200 \\
\hline 9 & 4335 & 4725 \\
\hline 10.5 & 4555 & 5512 \\
\hline
\end{tabular}

Using the obtained values of saturation flow shown in Table 9, saturation flow models were generated with width alone as independent variable considering different width combinations, in order to have a comparison with Webster's model. A linear regression model was developed between the saturation flow in $\mathrm{pc} / \mathrm{hr}$ and the lane width and was found to be:

$S=522 * w ; R^{2}=0.74$

where, $S$ is the saturation flow in $\mathrm{pc} / \mathrm{hr}$ and $w$ is the lane width in meters.

In a single lane width, the vehicle moves in a queue and the concept of lane based traffic followed in a car only condition can be simulated in a model developed for heterogeneous traffic. For a lane of width up to $7.0 \mathrm{~m}$, there is not much variation in saturation flow. In the model, the vehicles entered the simulation stretch as the simulation clock time equals the cumulative headway. There is less chance of having parallel arrival of vehicles at the start of the simulation stretch. Hence as width increases, there may be the chance of slight variation in saturation flow.

As per Highway Capacity Manual (2010), the base saturation flow is 1900 passenger car/ $\mathrm{hr}$, which is calculated for a lane width of 3.66 $\mathrm{m}$ having no right turning, no left turning and no heavy vehicles. The saturation flow for the ideal condition using the developed model given in Eq. (4) is 1911 passenger car/hr which shows close agreement with the HCM values. 


\section{ii) Mixed traffic condition}

Using the developed simulation model, experiments were conducted to study the various characteristics under mixed traffic flow. The experiments were conducted for various input values such as varying the approach widths, traffic volume, percentage composition, etc. Simulation runs were made using the developed model in a mixed traffic condition by varying the traffic composition of each vehicle type between 0 to $100 \%$ and also by varying the carriageway widths, keeping the widths at
$3.5,5.5,7,9$ and $10.5 \mathrm{~m}$. Cycle time of 120 seconds was adopted.

The saturation flow of the signalised intersection approach is taken as output from the simulation run for various traffic composition and approach widths. Using the simulation results, a linear multiple regression analysis has been made in order to estimate the saturation flow in veh/ $\mathrm{hr}$ as a function of the proportion of two_wheeler, three wheeler, car, bus and width. The model predicts saturation flow in vehicles/ hour. The model is shown in Eq. (5):

$S=61.016$ two_wheeler +32.2 three_wheeler +7.638 car -50.489 bus $+312.55 w$

where $S$ is the saturation flow in veh/hr, two wheeler, three wheeler, car, bus, $w$ is proportion of two wheelers, proportion of three wheelers, proportion of car, proportion of bus and width respectively. Table 10 shows the regression statistics.

\section{Table 10}

Saturation Flow Model Regression Statistics

\begin{tabular}{|c|c|c|c|}
\hline Variables & Coefficient & t-values & \multirow{2}{*}{$\mathbf{R}^{2}$} \\
\hline width & 312.556 & 8.693 & \\
\cline { 1 - 3 } two_wheeler & 61.016 & 17.840 & \multirow{2}{*}{0.96} \\
\cline { 1 - 3 } three_wheeler & 32.220 & 8.196 & \\
\hline car & 7.638 & 1.782 & \\
\hline bus & -50.489 & -6.211 & \\
\hline
\end{tabular}

The obtained $R^{2}$ value of the model gives a higher value, which shows that it is statistically significant. The higher t-values also show the significance of the model.

Thus the above model could be adopted for estimating saturation flows. For a $100 \%$ car condition, for a road width of $3.66 \mathrm{~m}$, saturation flow obtained from the model in a mixed traffic condition was $1908 \mathrm{pc} / \mathrm{hr}$ as against $1900 \mathrm{pc} / \mathrm{hr}$ of HCM.

\section{Conclusion}

This paper describes the various steps involved in the simulation processes such as vehicle generation, vehicle placement, vehicle movement, vehicle accumulation and vehicle dissipation. A flexible micro simulation traffic model TRAFFICSIM, written using object oriented programming (OOP) language was developed for simulating mixed traffic condition at signalized intersection. The development of such a traffic micro simulation model TRAFFICSIM based on a program written in $\mathrm{C} \#$ programming language is also explained in this paper. Micro simulation helps in creating more to realistic scenario of traffic flow.

The validation of TRAFFICSIM model, based on traffic composition, average speeds, queue discharge and accumulation, indicates that the model is significantly replicating 
the field conditions. Hence this model is highly recommended for estimating various traffic parameters by varying each of the input parameters and is very reliable for studying various characteristics in a mixed traffic condition. As an application of the simulation model, saturation flow models are developed for $100 \%$ car only condition and mixed traffic condition. A saturation flow of $1911 \mathrm{pc} / \mathrm{hr}$ was obtained for a lane width of $3.66 \mathrm{~m}$ in a homogeneous traffic condition and $1908 \mathrm{pc} / \mathrm{hr}$ in a mixed traffic condition, shows close agreement with the HCM values. Thus justifies the applicability of the model for both homogeneous and mixed traffic scenarios.

\section{References}

Arasan, V.T.; Arkatkar, S.S. 2010. Microsimulation study of effect of volume and road width on PCU of vehicles under heterogeneous traffic, Journal of Transportation Engineering, ASCE. DOI: http://dx.doi. org/10.1061/(ASCE)TE.1943-5436.0000176, 136(12): 1110-1119.

Arasan, V.T.; Dhivya, G. 2010. Methodology for Determination of Concentration of Heterogeneous traffic, Journal of Transportation Systems Engineering and Information Technology, 10(4): 50-61.

Arasan, V.T.; Koshy, R.Z. 2005. Methodology for Modeling Highly Heterogeneous Traffic Flow, Journal of Transportation Engineering, ASCE. DOI: http://dx.doi. org/10.1061/(ASCE)0733-947X(2005)131:7(544), 131(7): 544-551.

Arasan, V.T.; Krishnamurthy, K. 2008. Study of the Effect of Traffic Volume and Road Width on PCU Values of Vehicles Using Microscopic Simulation, Journal of the Indian Roads Congress, 69(2): 133-149.
Arasan, V.T.; Vedagiri, P. 2006. Estimation of saturation flow of heterogeneous traffic using computer simulation. In Proceedings of the 20th European Conference on Modelling and Simulation, ECMS.

Banks, J.; Carson II, J.S.; Nelson, B.L.; Nicol, D.M. 2000. Discrete-Event System Simulation. Prentice Hall of India Private Ltd., New Delhi.

Drew, D.R. 1968. Traffic Flow Theory and Control. Mc Graw Hill Company, New York.

Gates, T.J.; Noyce, D.A.; Laracuente, L. 2007. Analysis of Dilemma Zone Driver Behavior at Signalized Intersections, Transportation Research Board, Annual Meeting CD-ROM, Paper No. 07-3351.

Gowri, A.; Sivanandan, R. 2008. Evaluation of left turn channelization at a signalized intersection under heterogeneous traffic conditions, Transport. DOI: http:// dx.doi.org/10.3846/1648-4142.2008.23.221-229, 23(3): 221-229.

Hossain, M. 2001. Estimation of saturation flow at signalized intersections in developing cities: a micro-simulation modeling approach, Transportation Research Part A: Policy and Practice. DOI: http://dx.doi. org/10.1016/S0965-8564(99)00050-6, 35(2): 123-141.

Indian Roads Congress. 1994. Guidelines for the Design of At-Grade Intersections in Rural and Urban Areas-Special Publication 41. IRC, New Delhi, India.

Isaac, K.P. 1995. Studies on mixed traffic flow characteristics under varying composition, Ph.D. thesis, Bangalore University.

Isaac, K.P.; Veeraragavan, A. 1995. Headway distributions as an input to simulation models under mixed traffic flow. In Proceedings of the National Seminar on Emerging trends in Highway Engineering, Bangalore, T 2.1-2.14. 
Keller, E.L.; Saklas, J.G. 1984. Passenger Car Equivalents from Network Simulation, Journal of Transportation Engineering. DOI: http://dx.doi.org/10.1061/ (ASCE)0733-947X(1984)110:4(397), 110(4): 397-411.

Mallikarjuna, Ch.; Rao, K.R. 2006. Area Occupancy Characteristics of Heterogeneous traffic, Transportmetrica. DOI: http://dx.doi. org/10.1080/18128600608685661, 2(3): 223-236.

Radhakrishnan, P.; Mathew, T.V. 2011. Passenger car units and saturation flow models for highly heterogeneous traffic at urban signalised intersections, Transportmetrica. DOI: http://dx.doi. org/10.1080/18128600903351001 7(2): 141-162.

Transportation Research Board. 2010. Highway Capacity Manual. National Research Council, Washington, D.C.

Webster, F.V.; Cobbe, B.M. 1966. Traffic Signals. Technical Paper 56. HMSO, London. 